\title{
Síndrome de Burnout em profissionais de saúde da linha de frente contra a Covid-19
}

\author{
Burnout Syndrome in frontline health care professionals against Covid-19 \\ El Síndrome de Burnout en los profesionales de la salud de primera línea contra el Covid-19
}

\section{Resumo}

Objetivo: Identificar a Síndrome de Burnout em profissionais de saúde da linha de frente contra a Covid-19. Metodologia: Estudo transversal realizado com 48 profissionais de saúde que atuaram diretamente na linha de frente contra a Covid -19 há mais de 30 dias, num hospital do interior de Minas Gerais. Utilizou-se a escala Oldenburg Burnout Inventory (OLBI), para avaliação da presença da Síndrome de Burnout. Resultados: 54,2\% dos profissionais apresentaram sinais da Síndrome de Burnout, 27,1\% esgotamento profissional e 18,8\% não apresentavam sinais de esgotamento profissional. A maioria dos profissionais que apresentaram sinais síndrome são do sexo feminino e não estão em um relacionamento conjugal; não possuem outro vínculo empregatício, trabalham até 40 horas semanais, exercem cargo de nível médio, recebem até dois salários mínimos mensais, estão há mais de um ano na linha de frente e não apresentaram infecção pela Covid-19. Conclusão: Apesar de não se observar associação estatística significativa entre as variáveis sociodemográficas e profissionais e a ocorrência de sinais da Síndrome de Burnout, espera-se que os resultados do estudo em tela sirvam de subsídios para a formulação de políticas institucionais a fim de minimizar os efeitos negativos causados pela pandemia na saúde mental de seus colaboradores.

Palavras-chave: Esgotamento profissional; Covid-19; Esgotamento psicológico; Equipe de assistência ao paciente.

\begin{abstract}
Objective: To identify Burnout Syndrome in frontline health professionals against Covid-19. Methodology: Crosssectional carried out with 48 health professionals who worked directly on the front lines against Covid -19 for more than 30 days, in a hospital in the interior of Minas Gerais. The Oldenburg Burnout Inventory (OLBI) scale was used to assess the presence of Burnout Syndrome. Results: $54.2 \%$ of professionals showed signs of Burnout Syndrome, $27.1 \%$ professional burnout and $18.8 \%$ did not show signs of burnout. Most professionals who showed signs of syndrome are female and are not in a marital relationship; they have no other employment relationship, work up to 40 hours a week, hold a mid-level job, receive up to two minimum wages per month, have been on the front line for more than a year and have not been infected by Covid-19. Conclusion: Although there was no statistically significant association between sociodemographic and professional variables and the occurrence of signs of Burnout Syndrome, it is expected that the results of the study in question serve as subsidies for the formulation of institutional policies in order to minimize the negative effects caused by the pandemic on the mental health of its employees.
\end{abstract}

Keywords: Burnout, professional; Covid-19; Burnout, psychological; Patient care team. 


\begin{abstract}
Resumen
Objetivo: Identificar el Síndrome de Burnout en profesionales de la salud de primera línea frente la Covid-19. Metodología: Estudio transversal realizado con 48 profesionales de la salud que trabajaron directamente en primera línea contra Covid-19 durante más de 30 días, en un hospital del interior de Minas Gerais. Se utilizó la escala Oldenburg Burnout Inventory (OLBI) para evaluar la presencia del síndrome de Burnout. Resultados: el 54,2\% de los profesionales mostró signos de Síndrome de Burnout, el 27,1\% de Burnout profesional y el 18,8\% no mostró signos de Burnout. La mayoría de los profesionales que mostraron signos de síndrome son mujeres y no están en una relación matrimonial; no tienen otra relación laboral, trabajan hasta 40 horas a la semana, tienen un empleo de nivel medio, reciben hasta dos salarios mínimos al mes, llevan más de un año en primera línea y no han sido infectados por Covid19. Conclusión: Si bien no hubo asociación estadísticamente significativa entre variables sociodemográficas y profesionales y la ocurrencia de signos del Síndrome de Burnout, se espera que los resultados del estudio en cuestión sirvan como subsidios para la formulación de políticas institucionales con el fin de minimizar los efectos negativos causado por la pandemia sobre la salud mental de sus empleados.
\end{abstract}

Palabras clave: Agotamiento profesional; Covid-19; Agotamiento psicológico; Grupo de atención al paciente.

\title{
1. Introdução
}

Uma doença causando síndrome respiratória aguda grave se instalou em Wuhan, China, em dezembro de 2019 provocando infecções de fácil contágio provocado pelo novo Severe Acute Respiratory Syndrome Coronavírus 2 (SARS-CoV2). No mês de janeiro de 2020, a Organização Mundial da Saúde (OMS) declarou surto de infecção pelo Coronavírus (Covid19), e em 11 de março de 2020, uma pandemia (Santos \& Barbosa, 2020).

Diante do contexto de epidemias, os profissionais da saúde mostram um aumento da pressão psicológica, risco de morte e adoecimento. Durante esses acontecimentos, existe um aumento da carga horária de trabalho, exigindo jornadas de trabalho exaustivas, inclusive em outras instituições, o que pode gerar exaustão física, alta transmissibilidade hospitalar e exigem difíceis tomadas de decisões por parte dos profissionais respeitando a ética sobre os cuidados planejados e sua saúde (Santos et al., 2021).

A Síndrome de Burnout (SB) ou Síndrome do Esgotamento Profissional é caracterizada como um distúrbio que afeta a saúde emocional do profissional, causando despersonalização e diminuição da realização profissional e da rotina pessoal. É decorrente da exaustão profissional, como resposta de estresse crônico do seu trabalho, assim afetando com maior incidência trabalhadores que possuem maior contato com pessoas em seu dia a dia (Borges et al., 2021).

Dentre as profissões da saúde, a enfermagem é a que mais sofre por SB pois lida diretamente com o paciente e familiares, além de prestar assistência direta ao enfermo. A equipe de enfermagem encontra-se expostos aos riscos físicos, químicos e biológicos, o que faz com que o estresse na profissão seja desgastante, precisando assim de ações que visam seu bem estar físico, psíquico e social (Roseno et al., 2020).

Acredita-se que a exposição frente a SB tenha um aumento importante, pois com toda a problemática de episódios que experimentam ao longo de seu plantão, faz com que fiquem vulneráveis aos sinais e sintomas que descrevem a SB como: insônia, falta de apetite, ansiedade, problemas interpessoal e intrapessoal, medo, exaustão física.

A SB compreende como um problema de grande significância biopsicossocial, despertando grande preocupação por parte governamental, comunidade cientifica internacional, empresas e sindicatos, em razão do seu alto poder de consequências negativas causando transtornos mentais, ciclos viciosos, sendo responsável por afastamento no trabalho, aposentadoria precoce, licenças médicas, assim sendo reconhecida como agente patogênico no código previdenciário brasileiro desde 1999 (Ruback et al., 2018).

Nesse aspecto, o objetivo deste artigo foi identificar a Síndrome de Burnout em profissionais de saúde da linha de frente contra a Covid-19. 


\section{Metodologia}

Trata-se de um estudo transversal realizado com profissionais de saúde que atuaram diretamente na linha de frente contra a Covid -19, em um hospital do interior de Minas Gerais.

A amostra foi selecionada por conveniência, composta pela equipe multidisciplinar que atua no referido hospital há mais de 30 dias. Foi composta por 48 profissionais de saúde, dentre eles enfermeiros, técnicos de enfermagem, auxiliares de enfermagem, médicos, fisioterapeutas, psicólogos, nutricionistas e fonoaudiólogos.

A coleta de dados foi realizada nos meses de julho e agosto de 2021, por meio da utilização de dois instrumentos: um questionário sociodemográfico e profissional, a fim de traçar o perfil dos participantes; e a escala Oldenburg Burnout Inventory (OLBI), instrumento estruturado e validado para a população brasileira, contendo 13 questões, utilizada para mensurar a SB (Schuster \& Dias, 2018).

A avaliação da presença de sintomas relacionados à SB e a classificação dos profissionais foi realizada segundo a proposta de Peterson et al. (2008). Assim, escores médios $\geq 2,25$ na dimensão Exaustão (EE) e escores médios $\geq 2,1$ na Dimensão Distanciamento (DT) serão considerados altos e os indivíduos serão classificados conforme a seguinte associação: Sem Burnout (EE baixo e DT baixo); Com distanciamento (EE baixo e DT alto); Com esgotamento (EE alto e DT baixo); e Com Burnout (EE alto e DT alto).

Os dados coletados foram processados no software IBM® SPSS®, versão 21, para realização da análise descritiva e exploratória. Foram calculadas estatísticas descritivas por meio da utilização de medidas resumo de posição (média) e de variabilidade (amplitudes). Utilizou-se também, a análise bivariada por meio das medidas de associação com tabelas de contingência, além da aplicação do teste qui-quadrado de Pearson. Este trabalho considerou um nível de significância de 5\% $(\mathrm{p}<0,05)$.

A pesquisa seguiu as normas estabelecidas pela legislação vigente que rege sobre a realização de pesquisas com seres humanos, sendo aprovada pelo Comitê de Ética em Pesquisa do Centro Universitário do Cerrado Patrocínio (COEP/UNICERP) por meio do parecer $n^{\circ}$ 20211450ENF002. Os participantes do estudo consentiram participar do estudo mediante assinatura do Termo de Consentimento Livre e Esclarecido, sendo orientados previamente pelo pesquisador quanto aos objetivos da pesquisa e esclarecidos que não seriam submetidos a riscos ou prejuízos, e que poderiam desistir de participar do estudo a qualquer momento. Ainda, para minimizar a exposição dos participantes, eles foram identificados por meio de um código alfanumérico. Ademais, foi seguido o protocolo sanitário contra a Covid-19 valendo-se do uso de máscara obrigatório pelo pesquisador e participante, álcool em gel e distanciamento social.

\section{Resultados}

A amostra foi composta por 48 profissionais de saúde, sendo 24 (50\%) técnicos em enfermagem, 10 (20,8\%) enfermeiros, $4(8,3 \%)$ fisioterapeutas, $3(6,3 \%)$ médicos, $2(4,2 \%)$ fonoaudiólogos, $2(4,2 \%)$ nutricionistas, 2 (4,2\%) auxiliares de enfermagem e $1(2,1 \%)$ psicólogo.

A caracterização sociodemográfica e profissional da amostra está presente na Tabela 1. Destaca-se que a maioria $(72,9 \%)$ é do sexo feminino e não possui relacionamento conjugal (77,1\%). A média de idade encontrada foi de 34 anos, variando de 19 a 58 anos; $58,3 \%$ não possui outro vínculo empregatício; 70,8\% trabalha 40 horas semanais e 77,1\% trabalha há mais de um ano na linha de frente de combate à Covid-19. Quanto ao desenvolvimento da Covid-19, a maioria (64,6\%) relata não ter se infectado. Em relação à SB, 54,2\% apresentavam sinais da síndrome, 27,1\% esgotamento profissional e 18,8\% não apresentavam sinais de esgotamento profissional. 
Research, Society and Development, v. 11, n. 1, e33211124923, 2022

(CC BY 4.0) | ISSN 2525-3409 | DOI: http://dx.doi.org/10.33448/rsd-v11i1.24923

Tabela 1. Caracterização sociodemográfica e profissional da amostra (n = 48). Patrocínio - MG.

\begin{tabular}{lcc}
\hline & $\mathrm{n}$ & $\%$ \\
\hline Sexo & & \\
Masculino & 13 & 72,1 \\
Feminino & 35 & \\
Com relacionamento conjugal & 11 & 22,9 \\
Sim & 37 & 77,1 \\
Não & & 35,4 \\
Infecção por Covid-19 & 17 & 64,6 \\
Sim & 31 & 41,7 \\
Não & & 58,3 \\
Outro vínculo empregatício & 20 & 70,8 \\
Sim & 28 & 29,2 \\
Não & & \\
Carga horária semanal de trabalho & 34 & 22,9 \\
Até 40 horas & 14 & 77,1 \\
Mais de 40 horas & & \\
Tempo de trabalho na linha de frente & 11 & 68,8 \\
Até um ano & 37 & 27,1 \\
Mais de um ano & & 2,1 \\
Renda mensal* & 33 & 2,1 \\
Até 02 salários & 13 & 18,8 \\
De 03 a 05 salários & 1 & 27,1 \\
De 06 a 10 salários & 1 & 54,2 \\
Mais de 10 salários & 9 & \\
Classificação do estado de Burnout & 13 & 26 \\
Sem Burnout & & \\
Com esgotamento & & \\
Com Burnout & & \\
\hline & & \\
\hline
\end{tabular}

*Salário mínimo vigente no período da coleta de dados. Fonte: Dados da pesquisa (2021).

Não foi observada associação significativa entre as variáveis sociodemográficas e profissionais e a ocorrência de sinais da SB, conforme mostrado na Tabela 2. Ainda assim, a maioria dos profissionais que apresentaram sinais da Síndrome de Burnout são do sexo feminino e sem relacionamento conjugal; não possuem outro vínculo empregatício, trabalham até 40 horas semanais, exercem cargo de nível médio, recebem até dois salários mínimos mensais, estão há mais de um ano na linha de frente e não apresentaram infecção pela Covid-19. 
Tabela 2. Associação entre as variáveis sociodemográficas e profissionais e a ocorrência de sinais da Síndrome de Burnout (n = 48). Patrocínio - MG.

\begin{tabular}{|c|c|c|c|c|c|c|c|}
\hline & \multicolumn{2}{|c|}{ Sem Burnout } & \multicolumn{2}{|c|}{ Com esgotamento } & \multicolumn{2}{|c|}{ Com Burnout } & \multirow[b]{2}{*}{$\mathrm{p}$} \\
\hline & $\mathrm{n}$ & $\%$ & $\mathrm{n}$ & $\%$ & $\mathrm{n}$ & $\%$ & \\
\hline \multicolumn{8}{|l|}{ Sexo } \\
\hline Masculino & 2 & 22,2 & 3 & 23,1 & 8 & 30,8 & \multirow{2}{*}{0,60} \\
\hline Feminino & 7 & 77,8 & 10 & 76,9 & 18 & 69,2 & \\
\hline \multicolumn{8}{|c|}{ Com relacionamento conjugal } \\
\hline Sim & 2 & 22,2 & 4 & 30,8 & 5 & 19,2 & \multirow{2}{*}{0,81} \\
\hline Não & 7 & 77,8 & 9 & 69,2 & 21 & 80,8 & \\
\hline \multicolumn{8}{|l|}{ Infecção por Covid-19 } \\
\hline Sim & 1 & 11,1 & 8 & 61,5 & 8 & 30,8 & \multirow{2}{*}{0,40} \\
\hline Não & 8 & 88,9 & 5 & 38,5 & 18 & 69,2 & \\
\hline \multicolumn{8}{|l|}{ Outro vínculo empregatício } \\
\hline Sim & 3 & 33,3 & 5 & 38,5 & 12 & 46,2 & \multirow{2}{*}{0,49} \\
\hline Não & 6 & 66,7 & 8 & 61,5 & 14 & 53,8 & \\
\hline \multicolumn{8}{|l|}{ Nível do cargo ocupado } \\
\hline Ensino Médio & 4 & 44,4 & 6 & 46,2 & 16 & 61,5 & \multirow{2}{*}{0,35} \\
\hline Ensino Superior & 5 & 55,6 & 7 & 53,8 & 10 & 38,5 & \\
\hline \multicolumn{8}{|c|}{ Carga horária semanal de trabalho } \\
\hline Até 40 horas & 8 & 88,9 & 9 & 69,2 & 17 & 65,4 & \multirow{2}{*}{0,19} \\
\hline Mais de 40 horas & 1 & 11,1 & 4 & 30,8 & 9 & 34,6 & \\
\hline \multicolumn{8}{|c|}{ Tempo de trabalho na linha de frente } \\
\hline Até um ano & 4 & 44,4 & 2 & 15,4 & 5 & 19,2 & \multirow{2}{*}{0,14} \\
\hline Mais de um ano & 5 & 55,6 & 11 & 84,6 & 21 & 80,8 & \\
\hline \multicolumn{8}{|l|}{ Renda mensal* } \\
\hline Até 02 salários & 5 & 55,6 & 8 & 61,5 & 20 & 76,9 & \multirow{4}{*}{0,32} \\
\hline De 03 a 05 salários & 3 & 33,3 & 5 & 38,5 & 5 & 19,2 & \\
\hline De 06 a 10 salários & 1 & 11,1 & 0 & 0 & 0 & 0 & \\
\hline Mais de 10 salários & 0 & 0 & 0 & 0 & 1 & 3,8 & \\
\hline
\end{tabular}

*Salário mínimo vigente no período da coleta de dados. p: Nível de significância (p<0,05). Fonte: Dados da pesquisa (2021).

\section{Discussão}

A SB é reconhecida como uma doença ocupacional de alto risco entre os trabalhadores, assim causando prejuízos psíquicos podendo interferir na devida qualidade da assistência. Com o conhecimento dos fatores desencadeadores da síndrome é possível identificar os riscos que levam aos profissionais no sofrimento de tal patologia.

Dos profissionais que apresentaram SB, no estudo em tela, 69,2\% foram do sexo feminino. Dado corroborado por estudo de Roseno et al., (2020), em que revelou que o sexo feminino apresentou incidência de 77,1\%, em decorrência não só de seus cuidados no meio laboral, mas também no âmbito pessoal. Borges et al. (2021) e Borges et al. (2021) dizem que o sexo feminino apresenta um agravamento dos sintomas da SB pois pode se relacionar diretamente a profissão da enfermagem e desenvolver sintomas como depressão, ansiedade e angústia. Ainda Santos et al. (2021) reforçam que os agravos dos sintomas em mulheres aumentam por conta de seus hormônios sexuais, como o estradiol e a progesterona. Além disso, a psicologia diz que a mulher diferentemente do homem tem uma visão mais holística de ambiente, enquanto os homens analisam o ambiente e a si próprio de forma individual.

No presente estudo verificou-se que $80,8 \%$ das pessoas com SB não possui relação conjugal o que pode acarretar um aumento na sintomatologia da SB pois segundo Roseno et al., (2020) e Santos et al. (2021) estas pessoas não têm com quem dividir os problemas de vida. Além disso, os profissionais vivenciaram sentimentos de apoio inadequados, por parte da instituição de trabalho, população, mídia e familiares, o que causou decepção e desvalorização própria do profissional (Borges et al., 2021). Outro aspecto a ser considerado como fator desencadeante da SB em profissionais de saúde, neste período 
pandêmico, é o distanciamento social vivido decorrente da possibilidade de infectar-se ou contaminar familiares e amigos (Roberti et al., 2021).

Dentre os profissionais estudados, observa-se que nos profissionais com SB prevaleceu a escolaridade ensino médio (61,5\%), média de 40 horas semanais trabalhadas $(65,4 \%)$ e recebimento de até 2 salários mínimos (76,9\%). Estudo de Santos et al. (2021) se assemelha, pois relata a baixa remuneração dos profissionais no contexto da pandemia onde se teve um maior risco a vida e desvalorização para ganhos ínfimos. Ademais, Minayo e Freire (2020) relataram que os baixos salários dos profissionais, especialmente da enfermagem é alarmante, pois acarretam em consequências que beiram a insegurança de transporte e alimentar, sendo muitas vezes a realidade de quem mantêm o sistema de saúde em funcionamento, em plena pandemia.

No quesito infecção por Covid-19, a maioria dos profissionais não se infectou. Tal fato pode estar associado à intensificação nas medidas de precaução e biossegurança adotadas no contexto da pandemia (Silva et al., 2022; Oliveira, Lucas \& Iquiapaza, 2020). Soma-se a isso, a necessidade da realização de educação continuada sobre o tema (Teixeira et al.,2020).

Em relação à presença de outro vínculo empregatício, 46,2\% dos profissionais com SB possuem outro emprego. Estudo realizado por Rodrigues et al. (2017), no nordeste brasileiro, reforça tal ocorrência, em que 61,9\% dos profissionais apresentam duplo vínculo empregatício. Cabe salientar que a presença de mais vínculos empregatícios aumenta a carga horária semanal e diminui o tempo de descanso do profissional, o que favorece a ocorrência de desgaste físico e psíquico (Freitas \& Freire, 2020).

Segundo Ribeiro et al., (2020) apesar do protagonismo vivenciado pelos profissionais da saúde expostos as demandas de medo, exaustão, o papel de cuidador e a necessidade de um cuidado especializado e imediato para um significativo grupo de pessoas com sintomas da Covid - 19, estes profissionais permanecem em virtudes de cobrança e culpa de tentativas de organizar para conciliar as obrigações diárias e suas obrigações.

No presente estudo refere-se que dos profissionais com SB, 80,8\% trabalham há mais de um ano na linha de frente de combate à Covid-19, indicando maior exposição e tensão quanto à ocorrência do agravo. Borges et al. (2021) refere que esses profissionais que estão mais envolvidos na terapêutica e diagnóstico de pacientes com Covid - 19, estão altamente propensos a desenvolver angústias, pois esses profissionais não estão imunes a essa doença, e convivem com um cenário onde pacientes e colegas podem potencialmente evoluir a óbito.

\section{Conclusão}

A partir da realização do estudo, foi identificado que 54,2\% dos profissionais de saúde apresentavam sinais da SB, $27,1 \%$ esgotamento profissional e $18,8 \%$ não apresentavam sinais de esgotamento profissional.

Apesar de não se observar associação estatística significativa entre as variáveis sociodemográficas e profissionais e a ocorrência de sinais da SB, evidenciou-se que a maioria dos profissionais que apresentaram sinais da SB são do sexo feminino e sem relacionamento conjugal; não possuem outro vínculo empregatício, trabalham até 40 horas semanais, exercem cargo de nível médio, recebem até dois salários mínimos mensais, estão há mais de um ano na linha de frente e não apresentaram infecção pela Covid-19.

Espera-se que tais resultados sirvam de subsídios para a formulação de políticas institucionais a fim de minimizar os efeitos negativos causados pela pandemia na saúde mental de seus colaboradores.

Deve-se considerar algumas limitações na interpretação dos resultados deste estudo. A generalização dos achados é limitada, uma vez que a coleta de dados foi restrita a uma única instituição hospitalar, com tamanho amostral pouco expressivo, o que pode ter influenciado no resultado não significativo das associações entre as variáveis. Ademais, ressalta-se a 
importância da realização de novos estudos na temática a fim de investigar fatores relacionados à Síndrome de Burnout e o contexto pandêmico.

\section{Referências}

Borges, F. E. de, Borges Aragão, D., Borges, F. E., Borges, F. E., Sousa, A. S., \& Machado, A. L. (2021). Fatores de risco para a Síndrome de Burnout em profissionais da saúde durante a pandemia de COVID-19. Revista Enfermagem Atual In Derme, 95(33), e-021006. https://doi.org/10.31011/reaid-2020-v.94n.32-art.835

Borges, G. M., Maia, J. M., Xavier, P. O., Santos, A. B. dos R., Barbosa, C. C. M., Nogueira, V. F., \& Ito, A. M. (2021). O impacto da Síndrome de Burnout entre os profissionais de saúde no contexto da pandemia da Covid-19. Revista Eletrônica Acervo Enfermagem, 13 , e8375. https://doi.org/10.25248/reaenf.e8375.2021

Freitas, C. C., \& Freire, M. A. (2020). Síndrome de Burnout em enfermeiros trabalhadores de um hospital público de Natal, Rio Grande do Norte, Brasil. Revista Ciências em Saúde, 10 (2), 5-12. http://186.225.220.186:7474/ojs/index.php/rcsfmit_zero/article/view/937

Minayo, M., \& Freire, N. P. (2020). A pandemia agrava as desigualdades em saúde. Ciência \& Saúde Coletiva, 25(9), 3555-3556. https://doi.org/10.1590/1413-81232020259.13742020

Oliveira, A. C., Lucas, T. C., \& Iquiapaza, R. A. (2020). O que a pandemia da Covid-19 tem nos ensinado sobre adoção de medidas de precaução? Texto Contexto Enferm, 29: e20200106. https://doi.org/10.1590/1980-265X-TCE-2020-0106

Peterson, U., Demerouti, E., Bergström, G., Asberg, M., \& Nygren, A. (2008). Work characteristics and sickness absence in burnout and non burnout groups: A study of Swedish health care workers. International Journal of stress management, 15 (2), 153-172. https://doi.org/10.1016/0268-4012(95)90196-5

Ribeiro, L. M., Veira, T. A., \& Naka, K. S. (2020). Síndrome de burnout em profissionais de saúde antes e durante a pandemia da COVID-19. Revista Eletrônica Acervo Saúde, 12 (11), 1-10. https://acervomais.com.br/index.php/saude/article/view/5021.

Roberti, B. N., Murta, I. F., Albuquerque, I. C. A., Surdi, K. C., Assunção, P. H. S. S., \& Silva, Y. L. (2021). Síndrome de Burnout em trabalhadores de saúde da linha de frente durante a pandemia do COVID-19 no Brasil. Brazilian Journal Of Health Review, 4 (5), 21139-21150. https://www.brazilianjournals.com/index.php/BJHR/article/view/37025/pdf.

Rodrigues, C. C. F. M., Salvador, P. T. C. O., Assis, Y. M. S., Gomes, A. T. L., Bezerril, M. S., \& Santos, V. E. P. (2017). Estresse entre os membros da equipe de enfermagem. Rev Enferm UFPE on line, 11 (2), 601-608. https: 10.5205/reuol.10263-91568-1-RV.1102201715.

Roseno, D. A, Cavalcanti, J. R. L. P., \& Freire, M. A. M. (2020). Caracterização da síndrome de burnout em enfermeiros em municípios do interior do Estado da Paraíba - Brasil. Revista Ciência em Saúde, 10 (1), 01-08. http://186.225.220.186:7474/ojs/index.php/rcsfmit_zero/article/view/877.

Ruback, S. P., Tavares, J. M. A. B., Lins, S. M. de S. B., Campos, T. S., Rocha, R. G., \& Caetano, D. A. (2018). Stress and Burnout Syndrome Among Nursing Professinals Working in Nephrology: an Integrative Review. Revista De Pesquisa Cuidado é Fundamental Online, 10(3), 889-899. https://doi.org/10.9789/2175-5361.2018.v10i3.889-899

Santos, K. M. R, \& Barbosa, I. R. (2021). Depressão e ansiedade em profissionais de enfermagem durante a pandemia da COVID-19. Escola Anna Nery Revista de Enfermagem, 25, 1-15. http://www.scielo.br/scielo.php?script=sci_arttext\&pid=S1414-81452021000500201\&tlng=pt.

Santos, K. M. R., Galvão, M. H. R., Gomes, S. M., Souza, T. A., Medeiros, A. A., \& Barbosa, I. R. (2021). Depressão e ansiedade em profissionais de enfermagem durante a pandemia da covid-19. Escola Anna Nery Revista de Enfermagem, 25, 01-15. https://doi.org/10.1590/2177-9465-EAN-2020-0370

Silva, M. A. S., Lima, M. C. L., Dourado, C. A. R. O., Pinho, C. M., \& Andrade, M. S. (2022). Nursing professionals' biosafety in confronting COVID-19. Rev Bras Enferm., 75(Suppl 1), e20201104. https://doi.org/10.1590/0034-7167-2020-1104

Schuster, M. D. S., \& Dias, V. D. V. (2018). Oldenburg Burnout Inventory - validação de uma nova forma de mensurar Burnout no Brasil. Ciência \& Saúde Coletiva, 23, 553-562. https://doi.org/10.1590/1413-81232018232.27952015

Teixeira, C. F. S., Soares, C. M., Souza, E. A., Lisboa, E. S., Pinto, I. C. M., Andrade, L. R., \& Espiridião, M. A. A saúde dos profissionais de saúde no enfrentamento da pandemia de COVID-19. Ciência e Saúde Coletiva, 25 (9), 3465-3474. https://doi.org/10.1590/1413-81232020259.19562020 